\title{
LA GESTIÓN DE LA INNOVACIÓN TECNOLÓGICA EN MICROS, PEQUEÑAS Y MEDIANAS EMPRESAS DEL ESTADO DE QUERÉTARO. UN ESTUDIO EXPLORATORIO
}

\author{
Damaris Yunuen Mejía López*
}

\section{Mejía-López D.Y. La gestión de la innovación tecnológica en micros, pequeñas y medianas empresas del Estado de Querétaro. Un estudio exploratorio. Hitos de Ciencias Económico Administrativas 2014;20 (57): 57-67.}

\section{RESUMEN}

Objetivo: Identificar la existencia de procesos de gestión de la innovación tecnológica en las unidades de estudio seleccionadas del Estado de Querétaro, empleando 5 variables seleccionadas (vigilar, focalizar, capacitar, implantar y aprender).

Material y método: Los hallazgos se obtuvieron empleando un instrumento desarrollado con la metodología Thurstone, lo que le brinda fortaleza y confiabilidad, siendo una investigación exploratoria.

Resultados: Fue factible evaluar los cinco factores que permitirán identificar los diferentes niveles de gestión de la innovación tecnológica dentro de las micro, pequeñas y medianas empresas seleccionadas.

Conclusiones: Dentro de los hallazgos de la investigación, que factible visualizar que existen diferentes niveles de gestión de la innovación tecnológica entre las MIPyMEs, las áreas de oportunidad que tiene y la importancia de generar esquemas como el de proveedores confiables para impulsar su crecimiento.
Mejía-López D.Y. The management of technological innovation in micro, small and medium enterprises in the state of Querétaro. An exploratory study Hitos de Ciencias Económico Administrativas 2014;20 (57): 57-67.

\section{ABSTRACT}

Objective:Identify the existence of management processes of technological innovation in selected units Querétaro State study using 5 selected variables (monitor, target, train, deploy and learn).

Material and method: The findings were obtained using an instrument developed for this purpose with the Thurstone method which gives strength and driveability being an exploratory research .

Results: It was possible to evaluate the five factors that identify the different levels of management of technological innovation within the micro, small and medium enterprises selected.

Conclusions: Within the research findings is possible to visualize that there are different levels of management of technological innovation among MIPyMEs, areas of opportunity you have and the importance of generating schemes as reliable suppliers to boost growth.
Palabras clave: Gestión de la innovación tecnológica. Vigilar. Focalizar. Capacitar. Implantar. Aprender.
Key words: Management of technological innovation. Discipline. Focus. Train. Implement. Learn.

DIRECCIÓN PARA RECIBIR CORRESPONDENCIA: Correo electrónico: damaris.mejia7@gmaill.com

*Licenciada en administración. Estudiante del Programa de Maestría en Gestión de la Tecnología en la Universidad Autónoma de Querétaro. 
as reglas del juego para el logro de la competitividad han cambiado en forma radical con respecto al siglo que acaba de concluir. En este nuevo orden mundial, se imponen el aprovechamiento de las nuevas tecnologías, donde el conocimiento toma una dimensión preponderante y su gestión se convierte en un factor crítico para lograr prevalecer en un ambiente cada vez competitivo y exigente. En este orden de ideas, la gestión de la innovación tecnológica se yergue como una variable que se debe no solo de identificar, sino que además, se debe privilegiar si se desea participar en este concierto de las naciones, que divide cada vez más a los países capaces de desarrollar y aprovechar las nuevas tecnologías, obteniendo lo mejor de ellas a través de su capital intelectual y, aquellas que se ven forzadas a permanecer como mano de obra económica y proveedora de recursos naturales, debido a su incapacidad de coadyuvar al desarrollo de sus pobladores en parto por su falta de visión en la gestión de su tecnología.

\section{Antecedentes}

Si bien, resulta deseable el que las empresas sean capaces de desarrollar y aplicar tecnología propia, que les permita lograr avances y desarrollar modelos para dar respuestas eficientes a sus mercados, es una condición que se encuentra fuera del alcance de muchas de ellas, no solo por las inversiones que esto representa, sino además, por la falta de visión que existe al interior de las mismas, ya que muchas veces su mirada se encuentra fija en la sobrevivencia diaria y en la resolución de los problemas que enfrentan día a día. Es en este contexto que resulta fundamental el incorporar a las organizaciones el concepto de gestión de la innovación tecnológica, para coadyuvar en la adquisición, administración, desarrollo y/o fomento de las tecnologías que les permitan no solo sobrevivir, sino además, supervivir en el entorno cada vez más exigente del concierto empresarial. Lo antes mencionado, resulta aún más crítico cuando se trata de MIPyMEs, ya que en muchos casos su supervivencia depende de poder seguir el ritmo de las grandes empresas a las que sirven, mismas que les exigen cada vez más eficiencia y sincronía. En este sentido, se desea coadyuvar con estas empresas para que amplíen su visión y lograr competir no solo con calidad y precio, sino que anexen factores de alto valor como capacidad para diseñar soluciones a la medida para sus clientes, mejorar sus procesos integrales en la cadena de suministros, ofrecer servicios donde se brinden respuestas al cliente y a los clientes de sus clientes para ayudarlo a crecer y consolidarse al responder eficientemente a una demanda cada vez más diversificada. Sirva ello de antecedente para poder ingresar al presente trabajo de investigación.

\section{Objetivo}

Identificar la existencia de procesos de gestión de la innovación tecnológica en las unidades de estudio seleccionadas, empleando las 5 variables que a continuación se presentan:

\section{Variables}
1. Vigilar
2. Focalizar
3. Capacitar
4. Implantar
5. Aprender

\section{Marco teórico}

\section{Gestión de la innovación tecnología}

Para iniciar este camino, es importante partir del concepto que brinda Fundación Cotec (2001) el cual menciona que, una invención o idea creativa no es posible considerarla como innovación hasta que no se utiliza para cubrir una necesidad concreta. En este sentido, es posible identificar tres momentos o estados fundamentales en todo proceso de gestión de la innovación tecnológica:

Como primer punto la invención, como creación en si de una idea potencialmente generadora de beneficios comerciales, pero que no necesariamente es aterrizada en forma concreta en productos, procesos o servicios. Posteriormente la innovación, la cual consiste en la aplicación comercial de una idea, considerando que, innovar es convertir ideas en productos, procesos o servicios nuevos o mejorados que el mercado valora. Se trata de un hecho fundamentalmente económico que incrementa la capacidad de creación de riqueza de la empresa y, además, tiene grandes implicaciones sociales. Dicho concepto debe abarcar un amplio espectro de actividades de la empresa que presuponen un cambio substancial en la forma de hacer las cosas, tanto en lo que se refiere a los productos y servicios 
que ella ofrece, como a las formas en que los produce, comercializa u organiza.

Finalmente, la difusión, que supone dar a conocer a la sociedad la utilidad de una innovación. Es factible considerar a este el momento en el que un país percibe en forma verdadera los beneficios de la innovación.

En este orden de ideas, es posible comprender que el desarrollo económico de las organizaciones está estrechamente vinculado con su capacidad para realizar las tres actividades mencionadas y es en este punto donde surge la importancia de contar con una gestión adecuada de la innovación tecnológica, para obtener todo su potencial.

Para lograr una visión lo más integral posible sobre el tema, resulta importante revisar algunos conceptos básicos que permitan una mejor comprensión del contexto en que se desarrolla la investigación.

Considerando lo escrito por Frascati (1994), la ciencia puede ser vista como un método de generación de conocimientos, sustentado en la observación y el experimento. En tanto la tecnología, es la aplicación de los conocimientos científicos para obtener beneficios dando como fruto las actividades científicas y tecnológicas, las cuales se conceptualizan como las actividades sistemáticas relacionadas estrechamente con la generación, mejoramiento, difusión y aplicación del conocimiento científico y tecnológico, éstas es posible dividirlas en dos categorías básicas:

Investigación y Desarrollo Experimental (IDE)

Educación y Enseñanza Científica y Técnica (EECyT) Se refiere a todas las actividades de educación y enseñanza a nivel posgrado, estudios especializados, capacitación y actualización posteriores y de otorgamiento de becas.

\section{Servicios Científicos y Tecnológicos (SCyT)}

Son todas aquellas actividades relacionadas con la investigación y el desarrollo experimental que contribuye a la generación, difusión y a la aplicación de los conocimientos científicos y tecnológicos.

Con todo ello y, en sintonía con la Fundación Cotec (2001), quien menciona que el concepto de tecnología resulta ambiguo y frecuentemente se le asocia a la tecnología solo con máquinas y aparatos que funcionan, marginando de esta forma los aspectos relacionados con el conocimiento, es necesario ampliar mucho más el concepto de tecnología para ver más allá de máquinas que nos resultan tangibles y vislumbrar que incluye el conocimiento práctico orientado a la acción y, en este contexto se puede comprender como la aplicación sistemática del conocimiento científico u otro conocimiento organizado a tareas prácticas, logrando con ello comprenderlo como un conocimiento cuya aplicación está orientada a un fin concreto, a resolver problemas de acción, y su objeto no es simplemente saber, sino actuar. Es un conocimiento que se tiene no sólo cuando uno sabe, sino cuando sabe cómo hacer las cosas. Por todo ello, la técnica es la capacidad de utilizar métodos, instrumentos y equipos específicos para obtener resultados prácticos, en tanto que la tecnología exige además, la comprensión profunda de las limitaciones y perspectivas de dichas habilidades $y$, la capacidad de mejora de las mismas, por lo que implica una capacidad de cambio y mejora del conocimiento no incluido en la técnica. Anexo a ello, la ciencia se asocia con el conocimiento básico, con conceptos más genéricos, universalmente aplicables, pero menos poderosos al ser menos específicos. Por lo que, al considerar esta afirmación, la transformación de la ciencia en tecnología requiere la focalización del conocimiento científico en una gama concreta de problemas, siendo en este punto que se comprende la importancia no solo de la gestión adecuada de la tecnología sino además, la correcta utilización del capital intelectual.

Todo ello nos permite derivar a un concepto que abarca el proceso de la gestión de la innovación tecnológica la cual según Roberts (citado en Cotec, 2001, p.25) es «la organización y dirección de los recursos, tanto humanos como económicos, con el fin de aumentar la creación de nuevos conocimientos, la generación de ideas técnicas que permitan obtener nuevos productos, procesos y servicios o mejorar los ya existentes y, la transferencia de esas mismas ideas a las fases de fabricación, distribución y uso».

Para fines de este trabajo, se considera que los elementos claves para esta gestión de la innovación tecnológica incluyen los elementos citados por la Fundación Cotec (2001) en la figura 1. 
Figura 1

Innovación Tecnológica

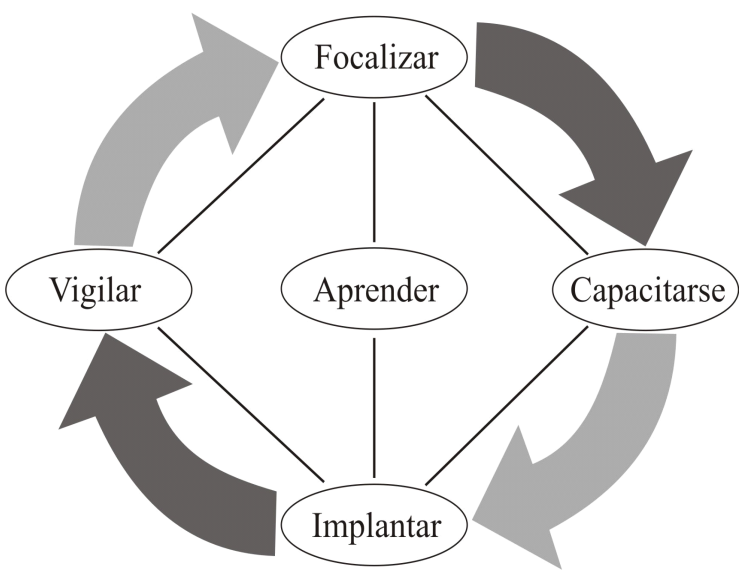

Fuente: Fundación Cotec, 2001.

En este orden de ideas y para esta investigación, se comprenderá a la gestión de la innovación tecnológica como el proceso que permite a las ideas intangibles, datos de mercado y tecnologías transformarse en un nuevo producto o proceso, ya que la investigación abarcará las empresas manufactureras, se considerará que para ellas la gestión de la innovación tecnológica se dará cuando en sus productos incluyan sus conocimientos y que éstos finalmente se vean reflejados en el diseño y especificaciones considerando una o varias de las etapas de su desarrollo, que pueden incluir desde el diseño de sus componentes, ensamble, desarrollo de prototipo final que logre pasar las pruebas hasta su aprobación para el logro de los objetivos para los que se diseñó. Este proceso resulta necesario para lograr aprender a gestionar la innovación tecnológica; básicamente se busca coadyuvar a lograr un aprendizaje organizativo cuyo objeto es reflexionar sobre los elementos anteriores, revisar experiencias tanto de éxito como de fallo, y aprender a realizar este proceso cada vez más eficientemente, logrando crear un aprendizaje significativo, mismo que permita dirigir el proceso de aprendizaje hacia lo que se ha denominado organizaciones que aprenden, lo cual puede dar frutos tales como lograr que los resultados y experiencias anteriores sean aprovechados de tal forma que sea posible capitalizar dicho conocimiento cuando se vuelvan a enfrentar condiciones similares en un futuro y la organización sea capaz de reconocer y anticiparse a los problemas.

Si bien, este es un proceso que puede resultar nuevo para una cantidad significativa de empresas mipem's, resulta fundamental para su supervivencia en un mercado cada vez más competitivo. Con la finalidad de presentar un esquema sencillo que sirva de punto de partida, para visualizar mejor este proceso, se presenta en la figura 2, propuesta por Amador y Márquez (2009).

\section{Figura 2 \\ Proceso de gestión en la innovación tecnológica}

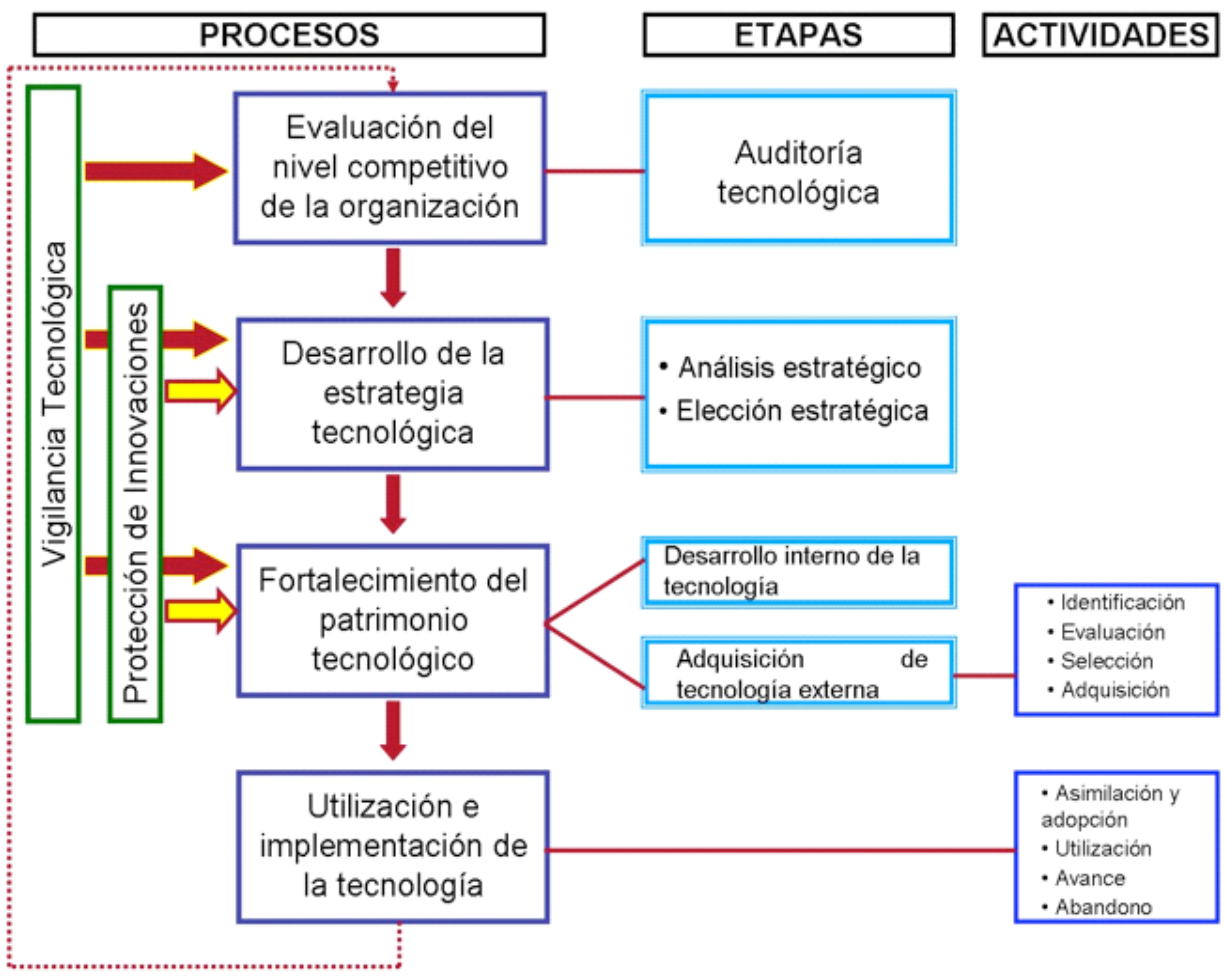

Fuente: Amador y Márquez, 2009. 
Resulta importante recordar que las empresas transnacionales inciden decisivamente en el desarrollo de capacidades de los proveedores locales por medio de las derramas tecnológicas, el impulso y las exigencias propias de la capacitación y las certificaciones, sin embargo, la condición es que el conocimiento diseminado por la red global debe ser absorbido por las empresas locales y eso depende solamente del desarrollo de capacidades que estas logren crear. Lograr los niveles de eficiencia que tienen las grandes empresas resulta fundamental para las pequeñas que las proveen, sin embargo, este parece un camino muy largo por andar ya que las diferencias

\section{Figura 3}

Valor agregado censal bruto por persona. (Miles de pesos)

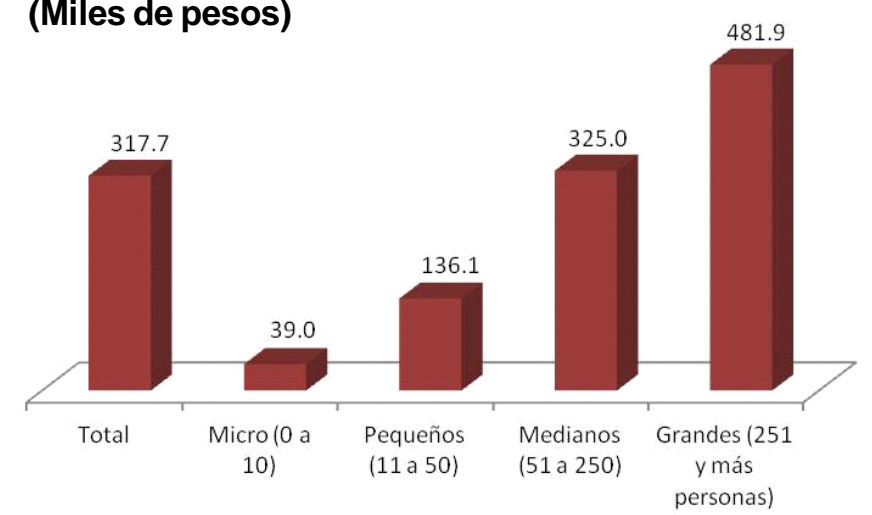

Micros, pequeñas y medianas empresas del Estado de Querétaro

Tal como se menciona en Censos Económicos INEGI (2009). El sector Manufacturero (el cual es el objeto de este estudio) acorde con el Sistema de Clasificación Industrial de América del Norte, México 2007 (SCIAN) está dividido en 21 subsectores, 86 ramas, 182 subramas y 292 clases de actividad. Las Industrias manufactureras están conformadas por unidades económicas dedicadas principalmente a la transformación mecánica, física o química de materiales o sustancias, con el fin de obtener productos nuevos. En este rubro también se consideran como parte de las manufacturas las actividades de maquila; el ensamble de partes y componentes o productos fabricados; la reconstrucción de maquinaria y equipo industrial, comercial, de oficina y otros; $y$ el acabado de productos manufacturados mediante el teñido, tratamiento calorífico, enchapado y procesos similares. Igualmente se incluye aquí la mezcla de materiales, como los aceites lubricantes, las resinas plásticas, las pinturas y los licores, entre otras.

Es factible visualizar su importancia económica en la figura 4.

Fuente: Fundación Cotec, 2001.

de eficiencia resultan en muchos casos muy grandes. Para visualizar esto se presentan los datos obtenidos de INEGI (2009) donde se menciona que el valor agregado censal bruto (valor que se adiciona a los insumos durante el proceso productivo) el cual el promedio que generó cada persona brindo un promedio en la micro empresa de 39,000 pesos anuales por persona, en cambio en la gran industria el promedio alcanzó 481900 pesos al año por persona, figura 3 .
Figura 4

Importancia de las industrias manufactureras en la economía 2008

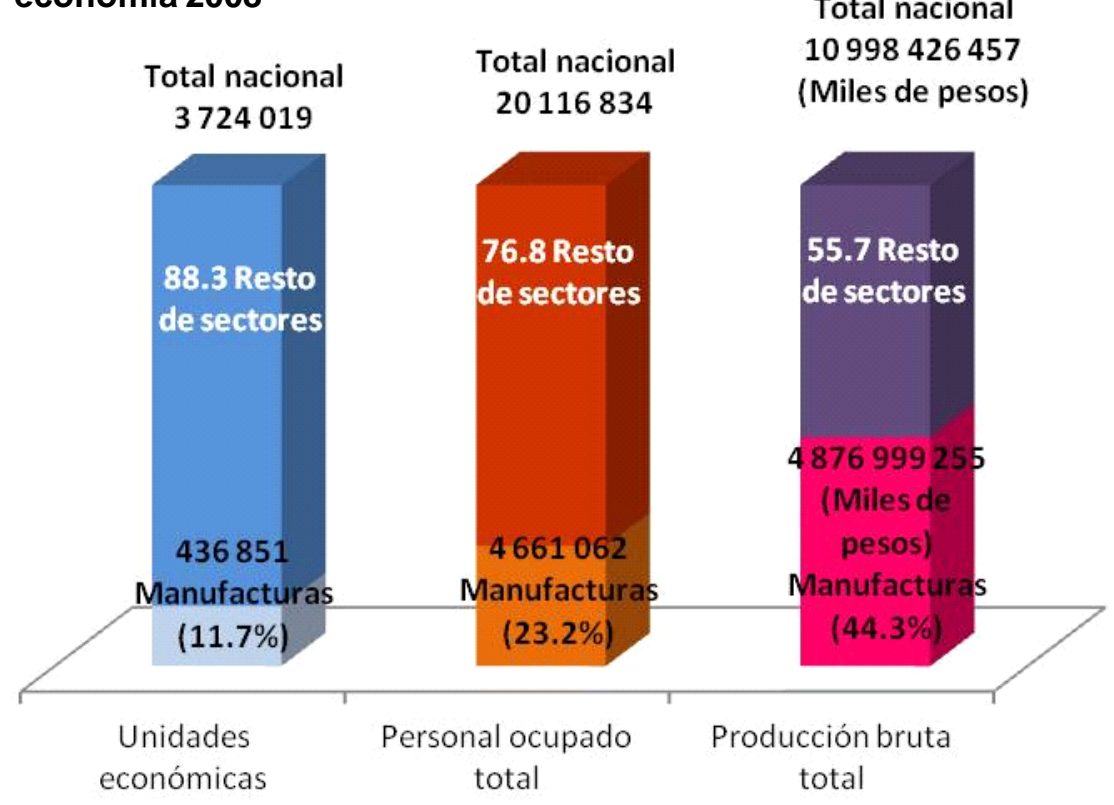

Fuente: Censos Económicos INEGI 2009 
Es factible ver la importancia en términos de unidades económicas y de personal ocupado por las mipem's en las siguientes gráficas.

\section{Población}

Se consideró como población a las unidades de estudio seleccionadas del Estado de Querétaro, las cuales incluirán empresas micro, pequeñas, medianas (mipem's), ubicadas en el Estado de Querétaro del área de manfactura (ver figura 5).

\section{Muestra}

Se empleará una muestra de sujetos tipos, ya que en este caso lo que importa es la riqueza, calidad y profundidad de la información por sobre la generalización, la cantidad y estandarización, (Hernández, 2003).

La muestra estuvo integrada por 182 mipem's ubicadas en Querétaro (64 micros, 60 pequeñas y 58 medianas).

Se considerarán para las unidades de estudio mipem's que se encuentren en la región centro del país, la cual teniendo como base la información de INEGI (2009), dicha región Centro del país (conformada por el Distrito

\section{Figura 5}

\section{Participación de la región en el total nacional}

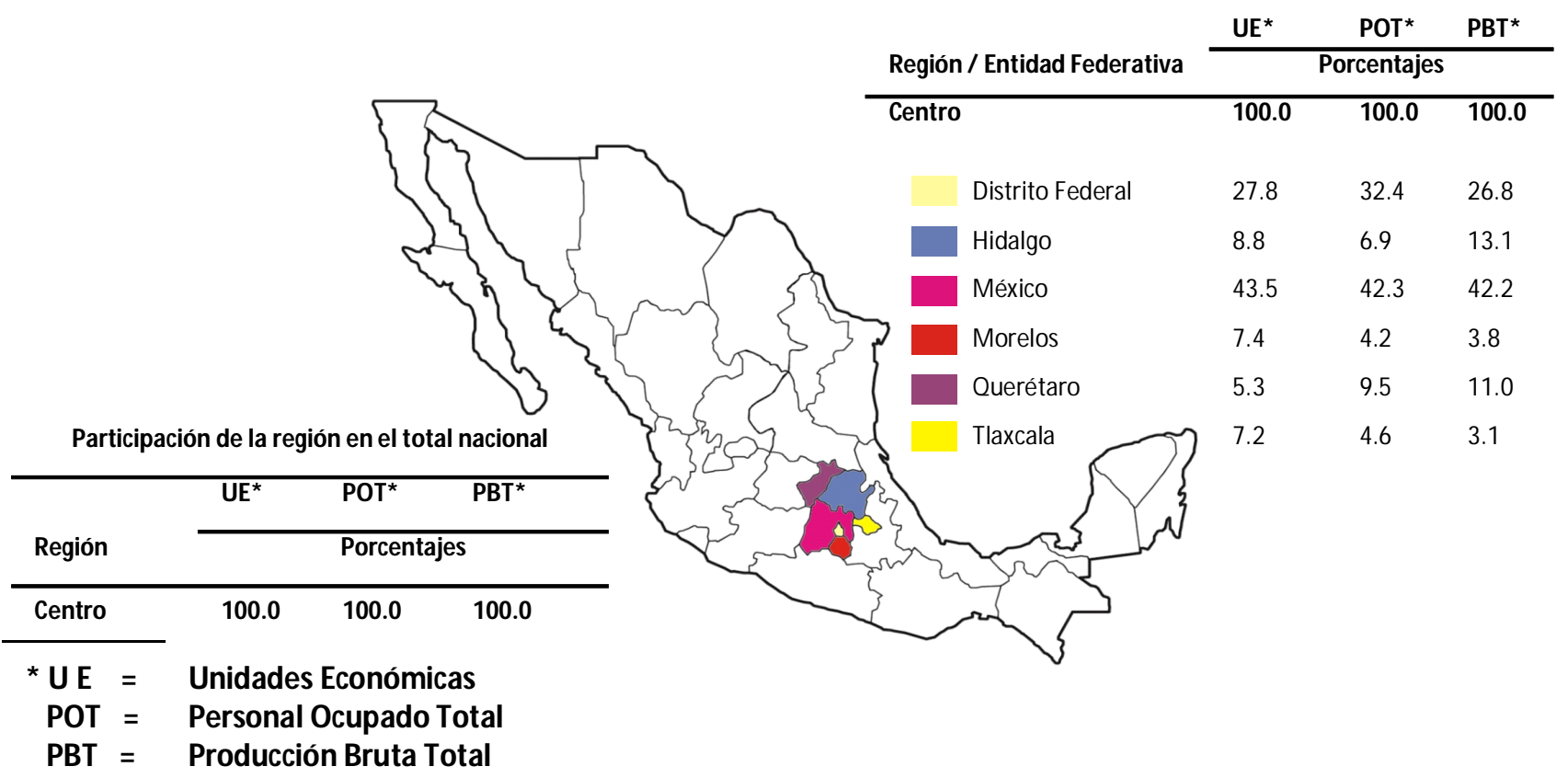

Federal, Hidalgo, México, Morelos, Querétaro y Tlaxcala) el estrato micro (0 a 10 personas) tuvo la mayor concentración de las unidades económicas con $90.7 \%$ y únicamente $2.5 \%$ de la producción bruta total; en cambio el estrato de las grandes (251 y más personas) contribuyó con $72.1 \%$ de la producción bruta total, $43.1 \%$ de personal ocupado total, con sólo $0.8 \%$ de unidades económicas (ver cuadro 1).

Las mipem's del estudio estarán consideradas por empresas que se encuentren conformadas por un máximo de 250 personas según el criterio de del Diario Oficial de la Federación del 30 de junio del 2009 presentado en el cuadro 2.

Es posible visualizar la importancia de dichas mipem's en el Estado de Querétaro en el cuadro que se presenta abajo ya que suman un total de 5814 unidades económicas en contraste con 110 que suman las empresas grandes.

\section{Marco metodológico}

\section{Diseño de la investigación}

El diseño de la investigación es No experimental, Transeccional, descriptivo. «Tiene como objetivo indagar la incidencia y los valores en que se manifiestan una o más variables o ubicar, categorizar y proporcionar una visión de una comunidad, un evento, un contexto, un fenómeno o una situación». Hernández, (2003, p.273).

Fuente: Censos Económicos INEGI 2009 
Tabla 1

Industrias manufactureras, región Centro

Unidades económicas por entidad, según tamaño (2008)

\begin{tabular}{|c|c|c|c|c|c|c|c|c|c|c|}
\hline \multirow{2}{*}{ Región/Entidad } & \multicolumn{2}{|c|}{ Total } & \multicolumn{2}{|c|}{ Micro } & \multicolumn{2}{|c|}{ Pequeños } & \multicolumn{2}{|c|}{ Medianos } & \multicolumn{2}{|c|}{ Grandes } \\
\hline & Absoluto & $\%$ & Absoluto & $\%$ & Absoluto & $\%$ & Absoluto & $\%$ & Absoluto & $\%$ \\
\hline Región Centro & 111121 & 100.0 & 100826 & 100.0 & 6898 & 100.0 & 2491 & 100.0 & 906 & 100.0 \\
\hline Distrito Federal & 30934 & 27.8 & 26491 & 26.3 & 3170 & 46.0 & 1013 & 40.7 & 260 & 28.7 \\
\hline Hidalgo & 9735 & 8.8 & 9106 & 9.0 & 445 & 6.5 & 128 & 5.1 & 56 & 6.2 \\
\hline México & 48357 & 43.5 & 44716 & 44.3 & 2279 & 33.0 & 944 & 37.9 & 418 & 46.1 \\
\hline Morelos & 8212 & 7.4 & 7832 & 7.8 & 277 & 4.0 & 77 & 3.1 & 26 & 2.9 \\
\hline Querétaro & 5924 & 5.3 & 5101 & 5.1 & 468 & 6.8 & 245 & 9.8 & 110 & 12.1 \\
\hline Tlaxcala & 7959 & 7.2 & 7580 & 7.5 & 259 & 3.8 & 84 & 3.4 & 36 & 4.0 \\
\hline
\end{tabular}

Fuente: Censos Económicos INEGI 2009

Tabla 2

Estratificación de empresas publicada en el Diario Oficial de la Federación 30 de junio de 2009

\begin{tabular}{|c|c|c|c|c|c|c|c|c|c|}
\hline \multirow[b]{3}{*}{ Sector } & \multicolumn{9}{|c|}{ Estratificacion } \\
\hline & \multicolumn{3}{|c|}{ Micro } & \multicolumn{3}{|c|}{ Pequena } & \multicolumn{3}{|c|}{ Medlana } \\
\hline & Personal & $\begin{array}{r}\text { Rango de } \\
\text { monto de } \\
\text { ventas } \\
\text { anuales } \\
\text { (mdp) }\end{array}$ & $\begin{array}{r}\text { Tope } \\
\text { maximo } \\
\text { combinado }\end{array}$ & Personal & $\begin{array}{r}\text { Rango de } \\
\text { monto de } \\
\text { ventas } \\
\text { anuales } \\
\text { (mdp) }\end{array}$ & $\begin{array}{r}\text { Tope } \\
\text { maximo } \\
\text { combinado* }\end{array}$ & Personal & $\begin{array}{r}\text { Rango de } \\
\text { monto de } \\
\text { ventas } \\
\text { anuales } \\
\text { (mdp) }\end{array}$ & $\begin{array}{r}\text { Tope } \\
\text { maximo } \\
\text { combinado }\end{array}$ \\
\hline Industria & De 0 a 10 & Hasta \$4 & 4.6 & De 11 a 50 & $\begin{array}{r}\text { Desde } \$ 4.01 \\
\text { hasta } \$ 100\end{array}$ & 95 & De 51 a 250 & $\begin{array}{r}\text { Desde } \$ 100.1 \\
\text { nasta } \$ 250\end{array}$ & 250 \\
\hline Comercio & De 0 a 10 & Hasta $\$ 4$ & 4.6 & De 11 a 30 & $\begin{array}{r}\text { Desde } \$ 4.01 \\
\text { hasta } \$ 100\end{array}$ & 93 & De 31 a 100 & $\begin{array}{r}\text { Desde } \$ 100.1 \\
\text { hasta } \$ 250\end{array}$ & 235 \\
\hline Servidios & De 0 a 10 & Hasta $\$ 4$ & 4.6 & De 11 a 50 & $\begin{array}{r}\text { Desde } \$ 4.01 \\
\text { hasta } \$ 100\end{array}$ & 95 & De 51 a 100 & $\begin{array}{r}\text { Desde } \$ 100.1 \\
\text { hasta } \$ 250\end{array}$ & 235 \\
\hline
\end{tabular}

Fuente: elaboración propia.

\section{Instrumento de medición}

El instrumento está compuesto por 18 ítems elaborados con metodología Thurstone, los cuales fueron aplicados a las unidades de investigación seleccionadas.
4.-Presentan procesos robustos y muestran evidencias de aplicación.
3.- Procesos en desarrollo con resultados.
2.- Procesos incipientes.

1.- Sin procesos o muestra de desarrollos.

\section{Dimensiones}

1.Vigilar. Esto incluye el análisis de mercado, tener perspectiva tecnológica, realización de benchmarking y el análisis de patentes.

2..Focalizar. Esto incluye las auditorias, la gestión de cartera, la evaluación de proyectos y la creatividad.

3.Capacitar. Los aspectos que se vinculan son la gestión de derechos de la propiedad intelectual e industrial, la gestión de interfaces, la gestión de 
proyectos, el trabajo en red y el funcionamiento en equipo.

4.Implantar. Se consideran la gestión del cambio, el funcionamiento ajustado, el análisis de valor;

5.Aprender. Esto incluye la mejora continua y la evaluación medioambiental.

\section{RESULTADOS Y DISCUSIÓN}

Es factible observar como las empresas medianas presentan un gran énfasis en el aspecto de vigilancia (figura 6), ya que presentaron procesos robustos y evidencias que mostraron sobre la aplicación de los cuatro factores considerados llegando a un promedio de $76.72 \%$ en comparación con las pequeñas empresas que solo obtuvieron un $31.66 \%$ y las micro empresas solo obtuvieron un $6.25 \%$ en dichos factores. En este orden de ideas, el $74.21 \%$ de las micro empresas que formaron parte de la muestra no presentaron procesos ni muestras de tener proyectos para desarrollar algunos de los cuatro factores investigados.

Sin duda, las exigencias que enfrentan día a día los empresarios los obligan a vivir resolviendo los problemas emergentes, sin embargo, resulta fundamental el lanzar la vista al futuro inmediato para buscar anticipar en la medida de lo posible los nuevos requerimientos y lograr un esquema que permita obtener ventajas competitivas de las innovaciones tecnológicas que aparecen en forma incesante y pueden cambiar del todo las necesidades del mercado.

Un aspecto relevante es el análisis de patentes ya que al permanecer atentos a este elemento brindara un «vistazo» al futuro y aunado con una política de perspectiva tecnológica fuerte ayudara en la realización de análisis de mercado y la oportuna realización del benchmarking necesario para permanecer dentro del mercado en forma exitosa.

Es factible observar como las empresas medianas brindan una gran importancia al aspecto de la Focalización (figura 7) ya que presentaron procesos robustos y evidencias donde mostraron la aplicación de los cuatro factores considerados llegando a un promedio de $96.98 \%$ en comparación con las pequeñas empresas que solo obtuvieron un $39.58 \%$ y las micro empresas solo obtuvieron un $28.12 \%$ en dichos factores. En este orden de ideas, el $53.51 \%$ de las micro empresas que formaron parte de la muestra no presentaron procesos ni muestras de tener proyectos para desarrollar algunos de los cuatro factores investigados

Durante la realización de la recolección de datos fue posible comprender que gran parte del énfasis que hacen las medianas empresas en torno a estos cuatro

\section{Figura 6 Vigilar}

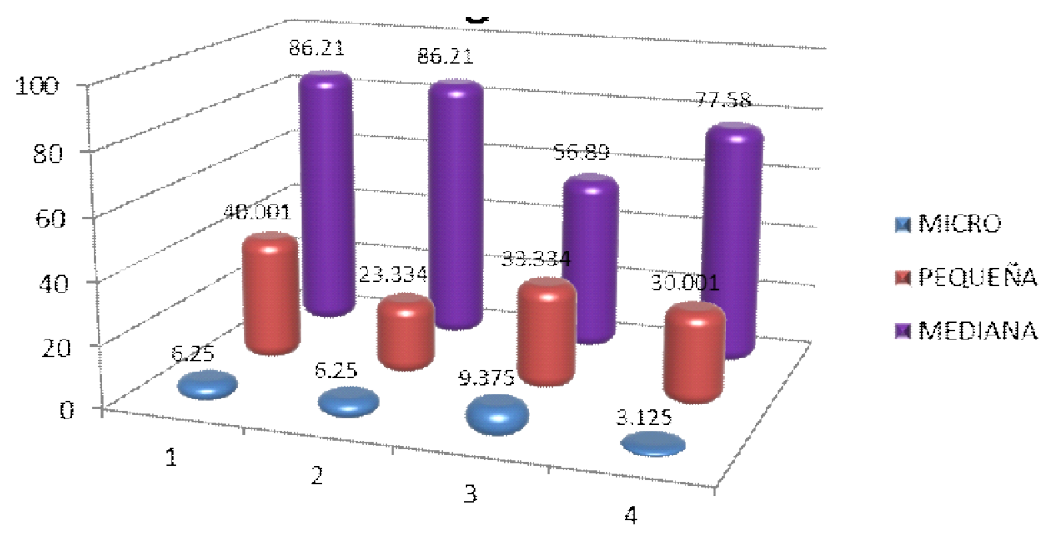

Fuente: Elaboración propia.

\section{Figura 7 \\ Focalizar}

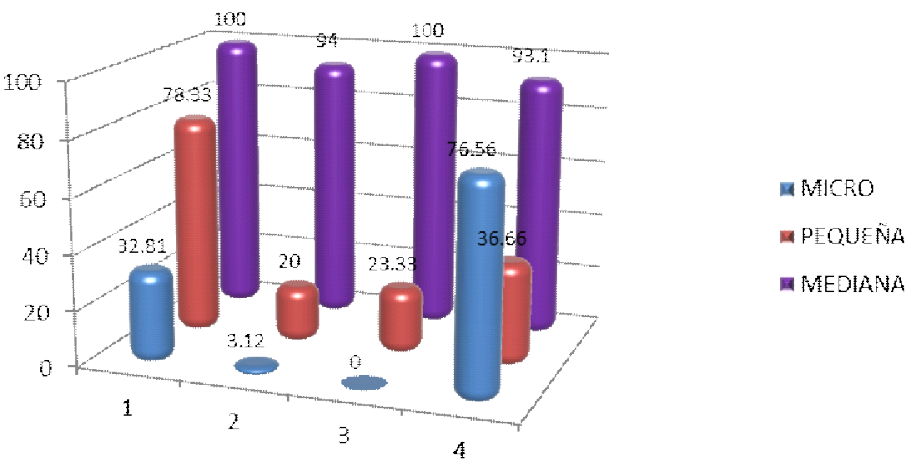

Fuente: Elaboración propia. 
elementos ha sido impulsado por su relación comercial con las grandes empresas a las que sirven ya que son ellas quienes realizan o les solicitan realizar los mismos y los visualizan en general como un área de oportunidad. Por contrapartida, en las micro empresas estos factores no solo son escasos, sino que son vistos como «necesidades que hay que cumplir» siendo más bien procesos a «librar».

Resulta factible observar como las empresas medianas imprimen una gran importancia en lo relativo a la Capacitación (ver figura 8) ya que presentaron tanto procesos robustos como evidencias de la aplicación de los cinco factores considerados llegando a un promedio de $98.62 \%$ en comparación con las pequeñas

\section{Figura 8}

Capacitar

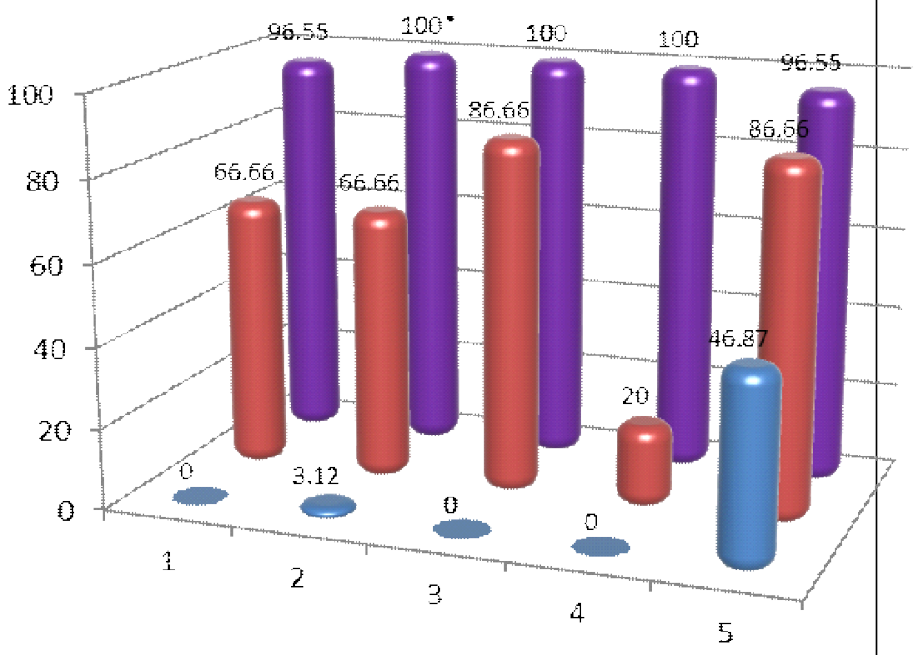

Fuente: Elaboración propia

\section{Figura 9 Implantar}

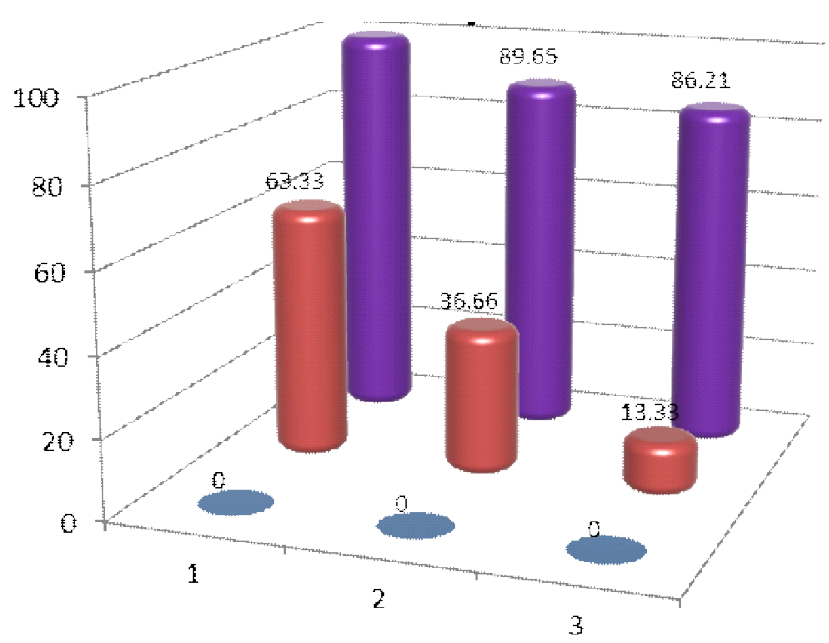

MCRO

G PEQUENAA

M MEDIAAA

empresas que solo obtuvieron un $65.33 \%$ y las micro empresas solo obtuvieron un $10.00 \%$ en dichos factores. En este orden de ideas, el $72.81 \%$ de las micro empresas que formaron parte de la muestra no presentaron procesos ni muestras de tener proyectos para desarrollar algunos de los cinco factores investigados.

Resulta revelador como las medianas empresas se han involucrado en forma importante en los temas relativos a la capacitación siendo impulsadas no solo por las empresas a las que sirven, sino además, para cumplir con lo dispuesto por la STPS y organismos tanto certificadores como verificadores. Mención especial es que en forma reiterada al entrar en estos factores se mencionó que muchas veces se aprende por «malas experiencias» ya que, debido a falta de información se habían enfrentado procesos derivados de la propiedad intelectual e industrial. Por contrapartida, en un número importante de las micro empresas encuestadas la capacitación sigue siendo vista como un gasto y los aspectos evaluados en este trabajo no estaban dentro de su planeación inmediata, ya que se consideraban más bien cursos para el desarrollo de habilidades manuales y de operación.

Es factible observar como las empresas medianas presentan un gran énfasis en lo relativo a implantar ya que presentaron procesos robustos y evidencias que mostraron la aplicación de los tres factores considerados llegando a un promedio de $91.95 \%$ en comparación con las pequeñas empresas que solo obtuvieron un 37.77 $\%$ y las micro empresas solo obtuvieron un $0 \%$ en dichos factores. En este orden de ideas, el $89.58 \%$ de las micro empresas que formaron parte de la muestra no presentaron procesos ni muestras de tener proyectos para desarrollar algunos de los tres factores investigados.

Fuente: Elaboración propia 
En lo relativo a la implantación las empresas medianas se encuentran involucradas en forma casi total ya que gran parte de sus contratos con las empresas grandes dependen de cumplir con los factores investigados encontrando que tienen áreas de oportunidad en lo relativo al funcionamiento ajustado el cual se consideró como el analizar todas las actividades de un proceso (dentro y fuera de la empresa) e identificar y eliminar todo desperdicio, conceptualizando éste último como aquellas actividades que no añaden ningún valor, de tal forma que sea posible gestionar en forma adecuada el cambio al interior de sus organizaciones y logren obtener un análisis de valor que coadyuve a la toma de decisiones. Un dato interesante en las micro empresas visitadas es que muchas de ellas carecen de la posibilidad de adaptarse a los cambios de diseños de sus productos y buscan ofrecer sus productos tal como los han realizado siempre buscando ganar los contratos con calidad y precio.

Es factible observar como las empresas medianas presentan un gran énfasis en lo relativo a Aprender (ver figura 10),ya que presentaron tanto procesos robustos como evidencias que mostraron la aplicación de los dos factores considerados llegando a un promedio de $100 \%$ en comparación con las pequeñas empresas que solo obtuvieron un $88.33 \%$ y las micro empresas solo obtuvieron un $21.87 \%$ en dichos factores. En este orden de ideas, el $46.87 \%$ de las micro empresas

\section{Figura 10}

Aprender

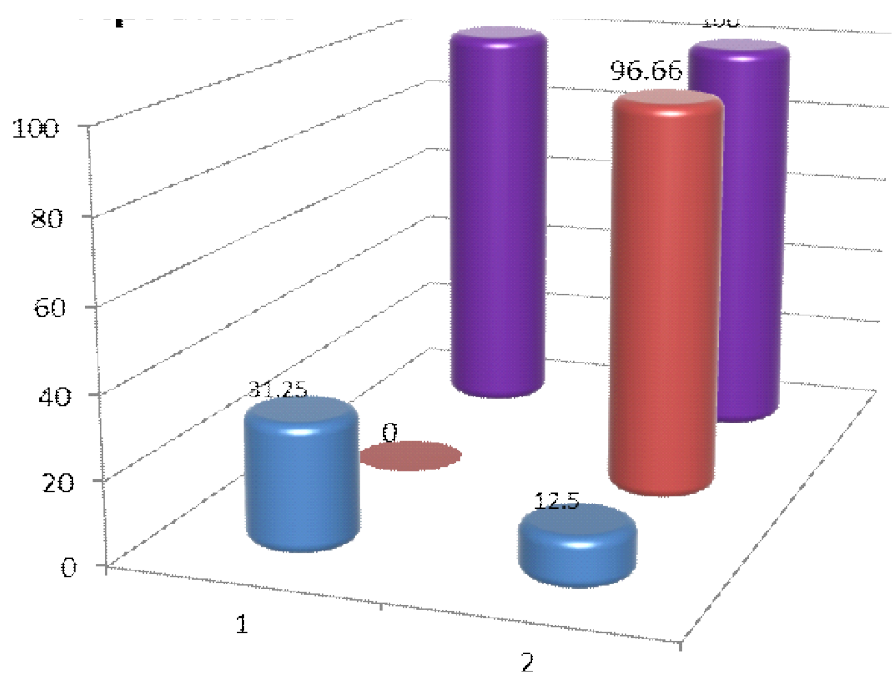

Fuente: Elaboración propia que formaron parte de la muestra no presentaron procesos ni muestras de tener proyectos para desarrollar algunos de los dos factores investigados.

Las empresas medianas se encuentran totalmente inmersas tanto en los procesos de mejora continua como en la evaluación medio ambiental, si bien, ambos factores han sido catapultados tanto por las exigencias de las empresas a las cuales proveen como por las autoridades respectivas resulta alentador ver que es campo de aplicación común para todas ellas. Por el contrario, en las micro empresas aún no existe un involucramiento al respecto de estos temas y en algunos temas, los visualizan como «trabas para producir».

\section{A manera de conclusiones}

Teniendo como base los hallazgos de la investigación es factible responder el problema de investigación al mencionar que:

Existen diferentes niveles en la aplicación de procesos para la gestión de la innovación tecnológica siendo estos escasos en las micros, moderados en las pequeñas y más desarrollados en las medianas empresas manufactureras seleccionadas del estado de Querétaro.

En este orden de ideas es posible aceptar la hipótesis planteada ya que:

Si existen procesos de gestión de la innovación tecnológica en las unidades de estudio seleccionadas.

Es importante remarcar que dichos procesos presentan diferentes niveles de desarrollo que van de los muy débiles en las micro empresas hasta niveles más altos en las empresas medianas seleccionadas en la muestra.

a MTHC

回 PEQUUENA

Fue posible lograr el objetivo ya que se identificó la existencia de procesos de gestión de la innovación tecnológica en las unidades de estudio seleccionadas empleando las cinco variables seleccionadas.

En gran medida las medianas empresas logran este nivel de desarrollo al verse envueltas en la dinámica y exigencias de las grandes empresas a quienes 
proveen y por las exigencias de las autoridades correspodientes.

Resulta deseable el fomentar esquemas que permitan desarrollar proveedores confiables en toda la cadena de suministros, de tal forma que, las micros y pequeñas empresas sean también involucradas en la dinámica y compromisos que adquieren las medianas empresas en la gestión de la innovación tecnológica. Si bien este proceso parece un tanto lejano (pues en las visitas realizadas a las micro empresas se pudo ver que existe un sentimiento de resistencia a innovar y una falta de visión sobre la importancia de gestionar dicha innovación) es una condición crítica no solo para la supervivencia de las mismas en un futuro no muy lejano, sino que además, se puede visualizar como una condición necesaria para el desarrollo del Estado y la sociedad en general.

Se recomienda que futuras investigaciones logren ampliar la base de empresas investigadas y se realicen estudios similares en otros Estados con la finalidad de obtener datos que permitan tener una visión más amplia de las áreas de oportunidad existentes.

\section{REFERENCIAS}

Amador, B., Márquez, A. (2009). Un modelo conceptual para gestionar la tecnología en la organización. A conceptual model to manage the technology in the organization. Revista espacios. 30(pp.6-8) http:// www.revistaespacios.com/a09v30n01/ 09300121.html

Censos Económicos (2009). Micro, pequeña, mediana y gran empresa: estratificación de los establecimientos: Censos Económicos 2009 / Instituto Nacional de Estadística y Geografía. México: INEGI, 2011.

Frascati Manual (1994). Measurement of scientific \& Technological Activities.

Fundación Cotec para la Innovación Tecnológica (2001). Innovación Tecnológica. Ideas Básicas. Colección Innovación Práctica. Madrid.

Hernández R, Fernández C, Baptista P. (2003), Metodología de la investigación, ( $3^{\underline{a}}$ ed.).

México: Mc Graw Hill

Manual de Oslo. (2005). Guía para la recogida e interpretación de datos sobre innovación. OCDE (Organización para la Cooperación y el Desarrollo Económico). Recuperado de: http://www.fia.cl/ Portals / O / U P P / D o c u m entos / Manual\%20de\%200slo.pdf

Revista internacional de ciencias sociales (2001). La dinámica del aprendizaje tecnológico en la industrialización. Linsu Kim (p 153-169) 